\title{
Transitions Between Electronic and Combustible Cigarettes: A Mixed Methods Analysis of Peer Interactions in an Online Community for Tobacco Cessation
}

\author{
Tavleen SINGH ${ }^{\mathrm{a}, 1}$, Nathan $\mathrm{COBB}^{\mathrm{b}}$, Trevor $\mathrm{COHEN}^{\mathrm{c}}$, and Sahiti MYNENI ${ }^{\mathrm{a}}$ \\ ${ }^{a}$ University of Texas Health Science Center School of Biomedical Informatics, \\ Houston, TX, USA \\ ${ }^{\mathrm{b}}$ Georgetown University Medical Center, Washington, DC, USA \\ ${ }^{\mathrm{c}}$ Biomedical Informatics and Medical Education, University of Washington, Seattle,
} WA, USA

\begin{abstract}
The growing popularity of e-cigarettes is a public health concern. There is an emerging need to understand the pathways between electronic and combustible modes due to the specialized nature of risks associated with each transition. Online social media has become the most dominant knowledge space for these evolving behaviors, and as such, can provide unique opportunities for modeling switching patterns. In this paper, we describe the utility of online peer interactions using qualitative inquiry and network visualizations using 500 messages to characterize (a) transition pathways and (b) psychosocial attributes as individuals contemplate and act on such transitions. Our results indicate that the E2A pathway is the most prevalent in e-cigarette-related transitions, where most of the individuals are in the "active e-cig use" stage. Perceived benefits and barriers are the most commonly held health beliefs, while counterconditioning and stimulus control behavior change processes are frequently manifested. Such insights can help in the design of personalized pathway-specific behavior change interventions.
\end{abstract}

Keywords. social media, behavior change, e-cigarettes, health beliefs

\section{Introduction}

Nicotine use is one of the world's largest public health problems killing more than 8 million people every year [1]. It is a significant risk factor for the development of cardiovascular diseases, respiratory problems, and various types of cancers leading to total economic costs of about US\$ 1.4 trillion annually [1]. While combustible cigarette smoking is the most common form of nicotine use worldwide, e-cigarettes have emerged as a new potential threat, with many scientific unknowns presenting challenges for public health surveillance and risk communications related to nicotine use [2]. Despite inconclusiveness about their short- and long-term health effects, e-cigarettes have remained increasingly popular among youth, young adults, and the general population [3]. Analyses of common use patterns of electronic and combustible nicotine products

\footnotetext{
${ }^{1}$ Corresponding Author: tavleen.kaur.ranjit.singh@uth.tmc.edu.
} 
emphasize a need to understand the transition pathways across nicotine intake modes [4]. For instance, most individuals use e-cigarettes to supplement rather than replace smoking (dual users), significantly increasing lung disease risk above smoking alone [5]. This highlights the specialized nature of risks associated with specific transition pathways between electronic and combustible modes of nicotine delivery. For individuals to achieve 'zero' or at least reduced nicotine dependence, it is essential to develop support interventions customized to the initial nicotine delivery mode and patterns of transition between delivery modes.

The role of online social media platforms in amplifying the popularity of e-cigarettes is well established [6]. These predominant information sources are digitally recorded and archived, providing us with invaluable datasets in the form of electronic traces of member interactions, which may help us understand the social drivers of transitions between multiple modes of nicotine intake (such as e-cigarettes to combustible cigarettes). Further, these data are seen as valuable, reliable, and often underutilized resources to detect nicotine use status [7]. However, very few studies have leveraged social media datasets to understand switching patterns between electronic and combustible delivery modes. Bridging these gaps, in this study, we will describe our qualitative coding efforts of peer interaction in an online community for tobacco cessation to characterize (a) an individual's current state of nicotine dependence (e.g., active smoker or abstinent) and (b) transition pathways between electronic and combustible modes of nicotine intake. Through this analysis, we aim to address the following research questions: a) what are the transition rates between electronic and combustible modes of nicotine delivery, as expressed in online social media platforms? and b) how do these transitions relate to theoretically-linked behavior change constructs?

\section{Methods}

QuitNet is one of the largest online health communities promoting tobacco cessation amongst its members [8]. The dataset used in this study consisted of 1478 de-identified forum messages (specifically mentioning e-cigarettes) spanning from 2009-2015, out of which 500 messages were randomly selected for manual coding. A single researcher coded the messages by performing line-by-line content analysis to identify the current behavioral state and transition pathway associated with every message. The behavior state and transition pathway codes were mutually exclusive. As illustrated in Figure 1, the current behavior state of "Active Dual use" and the transition pathway of "Dual to Ecigs (D2E)" was extracted from the sample message. After initial coding at the message level, the messages were further grouped based on member-level context (such as member ids and temporal attributes), which led to extrapolation of behavior states and transition pathways for the remaining of the messages. Deeper analysis guided by the Transtheoretical of change (TTM) [9] and Health Belief Model (HBM) [10] allowed us to identify the stage of transition (mutually exclusive codes: Contemplation, Preparation, Action, Maintenance) and underlying TTM processes (e.g., stimulus control, reinforcement management) and HBM constructs (e.g., perceived benefits, perceived barriers) which were not mutually exclusive codes. The definitions for TTM and HBM constructs are described here $[9,10]$. Messages which do not provide enough semantic context were coded as Not Applicable (NA). Further, network visualizations were generated to represent the multilevel inter-and intra-personal theoretical constructs underlying transition pathways as manifested in our analysis of online peer interactions. 


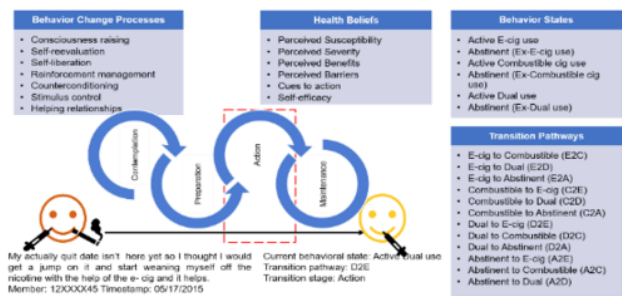

Figure 1. Qualitative coding schema

\section{Results and Discussion}

During the initial coding at the message level, we identified behavior states in 403 messages and found evidence of transition in 395 messages. Based on member-level context, we extrapolated the behavior states and transition pathways coding to 496 messages. The most prevalent behavior state as indicated by forum messages was abstinent (ex-combustible cigarette use) $(n=268)$, followed by active e-cigarette use $(n=124)$, abstinent (ex-dual use) $(n=49)$, abstinent (ex-e-cigarette use) $(n=33)$, active dual use $(n=13)$, and active combustible cigarette use $(n=9)$. The most prevalent transition pathway was C2A $(n=255)$, followed by E2A $(n=92)$ and C2E $(n=72)$. Several messages indicated that members had used e-cigarettes at some point in the past, but that did not eventually help them to quit, and they finally transitioned towards abstinence either by quitting "cold turkey" or using FDA-approved smoking cessation products (patches, lozenges) (D2A) ( $\mathrm{n}=53)$. Some messages also revealed the use of e-cigarettes to cope with the withdrawal symptoms $(\mathrm{A} 2 \mathrm{E})(\mathrm{n}=16)$, while few messages indicated drifting towards dual-mode to prepare for quits by substituting smoking needs with e-cigarettes $(\mathrm{C} 2 \mathrm{D})(\mathrm{n}=8)$. For further analysis, we focused on different transition pathways leading to and from e-cigarettes (including dual-modes) $(n=241)$. Table 1 indicates the readiness of members in each of the ascertained transition pathways. Interestingly, the majority of the messages in the A2E pathway were in the "Contemplation" stage, indicating how community members weigh the pros and cons of e-cigarettes, while a higher count of messages belonging to other pathways were in the "Action" stage, indicating the need for the informational ecosystem to serve as evidence-based decision support aids.

Table 1. Distribution of various behavior stages for different transition pathways.

\begin{tabular}{ccccc}
\hline Transition Pathway & Contemplation & Preparation & Action & Maintenance \\
\hline A2E $(\mathrm{n}=16)$ & 15 & 0 & 1 & 0 \\
C2D $(\mathrm{n}=8)$ & 0 & 1 & 7 & 0 \\
C2E $(\mathrm{n}=72)$ & 1 & 8 & 53 & 10 \\
D2A $(\mathrm{n}=53)$ & 1 & 4 & 38 & 3 \\
E2A $(\mathrm{n}=92)$ & 1 & 3 & 75 & 11 \\
& $(\mathrm{n}=18)$ & $(\mathrm{n}=16)$ & $(\mathrm{n}=174)$ & $(\mathrm{n}=24)$ \\
\hline
\end{tabular}

Our analysis revealed the manifestation of behavior change processes and health beliefs specific to transition pathways. As seen in Table 2, "consciousness raising" (CR) was common during the $\mathrm{A} 2 \mathrm{E}$ transition where members were seeking information to gain an understanding of the advantages and disadvantages of using e-cigarettes over combustible cigarettes, for example, "I just saw an ad for the e-cigs and was curious to see what people thought about them on here". "Self-reevaluation" (SR) was also populous during $\mathrm{A} 2 \mathrm{E}$ transition where members were appraising their quit efforts, and switching to e-cigarettes since they viewed it as a healthier version, for example, "I think 
I'd rather go to the e-cig than start back up smoking ciggies". The members transitioning from $\mathrm{C} 2 \mathrm{E}$ used "self-liberation" (SL) by committing to changing their behaviors, for example, "I'm working on starting my quit with the e-cig". "Reinforcement management" (RM) process was frequently utilized during C2E and E2A transitions where members engaged in appreciating themselves through tangible/virtual rewards and reinforcing self-talk, for example, "I feel like superman every time I put a checkmark on my calendar for the day before, indicating that I DID NOT smoke. You can be your own superhero in this". "Counterconditioning" (CC) was the most prevalent behavior change process across most transition pathways, where members discussed e-cigarettes (C2E, E2A, C2D transitions) and products such as nicotine gum, cinnamon sticks. (D2A). "Stimulus control" (SC) was evident in C2E, D2A, E2A pathways to prevent relapses, "My urge to smoke was through the roof that day but instead - I grabbed the e-cig out of my car (now using no-nicotine menthol) and took a hit off of that". Some members mentioned "helping relationships" (HR), where a friend suggested e-cigarette use.

Table 2. Distribution of TTM-specific behavior change processes for different transition pathways.

\begin{tabular}{cccccccc}
\hline Transition Pathway & CR & SR & SL & RM & CC & SC & HR \\
\hline A2E $(n=16)$ & 9 & 14 & 0 & 0 & 1 & 0 & 0 \\
C2D $(n=8)$ & 0 & 0 & 0 & 1 & 8 & 1 & 0 \\
C2E $(n=72)$ & 1 & 1 & 10 & 16 & 59 & 18 & 3 \\
D2A $(\mathrm{n}=53)$ & 0 & 1 & 4 & 5 & 21 & 23 & 1 \\
E2A $(\mathrm{n}=92)$ & 1 & 1 & 5 & 21 & 73 & 24 & 7 \\
& $(\mathrm{n}=11)$ & $(\mathrm{n}=17)$ & $(\mathrm{n}=19)$ & $(\mathrm{n}=43)$ & $(\mathrm{n}=162)$ & $(\mathrm{n}=66)$ & $(\mathrm{n}=11)$ \\
\hline
\end{tabular}

As seen in Table 3, the most prevalent belief was "perceived benefits" especially during E2A and C2E transitions. The members believed in the utility of e-cigarettes as a quit tool (e.g., "Many small-scale clinical trials have supported e-cigs as being $0.05 \%$ harmful compared to $100 \%$ harm done by smoking". Some members also reported their "perceived barriers" especially during D2A transitions, for example, "I think e-cigs are too closely related to an actual cig in the sense of holding it and inhaling it". "Perceived susceptibility" and "perceived severity" beliefs showed that some community members were aware of the harmful health effects associated with smoking cigarettes, which motivated them to change their current behaviors, for example, "My chances for getting cancer after 44 years of smoking are so high that I am not worried about that, the damage is done" and "Also, I just have this feeling that e-cigs aren't safe". Members exhibited other HBM constructs such as "self-efficacy" (e.g., "e-cig gave the confidence to quit") and "cues to action" (e.g., "Just a few weeks ago, I had an urge and it was stronger than usual - so I pulled out that e-cig with the non-nicotine cartridge".

Table 3. Distribution of constructs related to health beliefs for different transition pathways.

\begin{tabular}{ccccccc}
\hline $\begin{array}{c}\text { Transition } \\
\text { Pathway }\end{array}$ & $\begin{array}{c}\text { Perceived } \\
\text { Susceptibility }\end{array}$ & $\begin{array}{c}\text { Perceived } \\
\text { Severity }\end{array}$ & $\begin{array}{c}\text { Perceived } \\
\text { Benefits }\end{array}$ & $\begin{array}{c}\text { Perceived } \\
\text { Barriers }\end{array}$ & $\begin{array}{c}\text { Cues to } \\
\text { Action }\end{array}$ & $\begin{array}{c}\text { Self- } \\
\text { efficacy }\end{array}$ \\
\hline A2E $(\mathrm{n}=16)$ & 0 & 1 & 6 & 8 & 9 & 0 \\
C2D $(\mathrm{n}=8)$ & 1 & 0 & 5 & 2 & 2 & 1 \\
C2E $(\mathrm{n}=72)$ & 2 & 2 & 50 & 12 & 4 & 20 \\
D2A $(\mathrm{n}=53)$ & 4 & 10 & 5 & 40 & 11 & 5 \\
E2A (n=92) & 0 & 1 & 66 & 11 & 13 & 25 \\
& $(\mathrm{n}=7)$ & $(\mathrm{n}=14)$ & $(\mathrm{n}=132)$ & $(\mathrm{n}=73)$ & $(\mathrm{n}=39)$ & $(\mathrm{n}=51)$ \\
\hline
\end{tabular}

In summary, Figure 2 presents an overview of behavioral constructs in "Action stage" in C2E and E2A pathway-specific affiliation networks (edge width represents tie strength). 


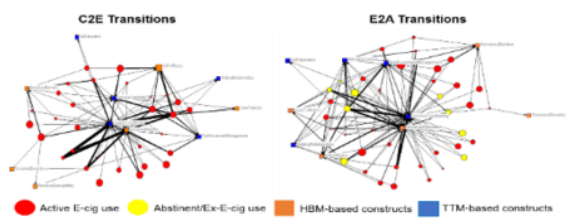

Figure 2. Affiliation networks specific to transition pathways between combustible and e-cigarettes.

Our work is not without limitations. Although randomly selected, the low number of annotated messages might have led to inaccurate behavior representations. Although we used sound theoretical frameworks, there may be subjective bias in the annotation due to a single coder. In future work, we will leverage computational language representations to achieve the scalability of our analysis to large scale datasets.

\section{Conclusions}

Our work highlights the utility of social media datasets in identifying behavioral states and transitions to better understand the psychosocial factors associated with pathways leading to and from electronic modes of nicotine delivery. This can have implications for designing pathway-specific, stage-responsive quit interventions to enable individuals to sustain healthier lifestyles with zero nicotine dependence.

\section{Acknowledgements}

Research reported in this publication was supported by the National Cancer Institute of the National Institutes of Health under Award Number 1R01LM012974-01A1. The content is solely the responsibility of the authors and does not necessarily rep-resent the official views of the National Institutes of Health.

\section{References}

[1] Tobacco. Available at: https://www.who.int/westernpacific/health-topics/tobacco, Accessed 2021 Jan 16.

[2] Public Health Consequences of E-Cigarettes [Internet]. Eaton DL, Kwan LY, Stratton K, editors. Washington (DC): National Academies Press (US); 2018, Accessed 2021 Jan 28.

[3] Dai H, Leventhal AM. Prevalence of e-Cigarette Use Among Adults in the United States, 2014-2018. JAMA. 2019 Sep 16;

[4] Baig SA, Giovenco DP. Behavioral heterogeneity among cigarette and e-cigarette dual-users and associations with future tobacco use: Findings from the Population Assessment of Tobacco and Health Study. Addict Behav. 2020 May;104:106263.

[5] Bhatta DN, Glantz SA. Association of E-Cigarette Use With Respiratory Disease Among Adults: A Longitudinal Analysis. Am J Prev Med. 2020 Feb;58(2):182-90.

[6] Payne JD, Orellana-Barrios M, Medrano-Juarez R, Buscemi D, Nugent K. Electronic cigarettes in the media. Proc (Bayl Univ Med Cent). 2016 Jul;29(3):280-3.

[7] Myneni S, Cobb NK, Cohen T. Content-specific network analysis of peer-to-peer communication in an online community for smoking cessation. AMIA Annu Symp Proc. 2017 Feb 10;2016:934-43.

[8] Cobb NK, Graham AL, Bock BC, Papandonatos G, Abrams DB. Initial Evaluation of a Real-World Internet Smoking Cessation System. Nicotine \& Tobacco Research. 2005 Apr 1;7(2):207-16.

[9] Prochaska JO, Velicer WF. The Transtheoretical Model of Health Behavior Change. Am J Health Promot. 1997 Sep;12(1):38-48.

[10] Champion VL, Skinner CS. The health belief model. In: Health behavior and health education: Theory, research, and practice, 4th ed. San Francisco, CA, US: Jossey-Bass; 2008. p. 45-65. 